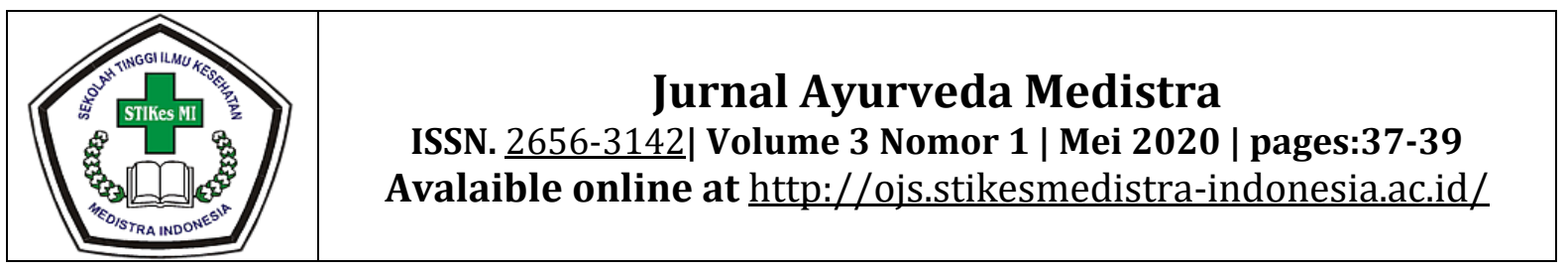

\title{
IMPLEMENTATION OF THERAPEUTIC DRUG MONITORING IN INDONESIA
}

\author{
Aluwi Nirwana Sani \\ Pharmacy Study Program S1 STIKes Medistra Indonesia, medistra@stikes.ac.id, 081212360165
}

\begin{abstract}
Therapeutic Drug Monitoring (TDM) is the clinical pharma cy practice of measuring plasma level of drugs. This service is required to optimize the drug therapy in individual patient and should be considered an adjunct to physician's clinical judgment in assessing the course and the effectiveness of treatment, especially in critically ill patients. Indonesia has not been developed this service. Therefore, this article discus the roles and function of a best practice TDM service and how can this service be implemented in Indonesia.
\end{abstract}

Keywords:Drug concentration, drug monitoring, patient, blood samples

\section{Introduction}

Therapeutic Drug Monitoring (TDM) involves measuring drug concentration in plasma, serum or blood. This measurement is used to individualize dosage so that drug concentrations can be maintained within target range. (1) Drug level at the site of action can not be measured, however the desired and adverse effect may correlate better with plasma or blood level than they do with dose. For a few drugs, concentration measurements are a valuable for drugs with no simple or sensitive measure of effect.

When there is a large inter-individual variation between dose and effect, in other word there is large pharmacokinetics variation, individual drug dose is difficult. This is particularly relevant for drugs with narrow therapeutic window or concentration-dependent pharmacokinetics. Similarly, variation in individual can occur over time for a range of reasons, with some drugs, for example critically ill patients, and TDM could be useful.

\section{Therapeutic Drug Monitoring Practice.}

TDM is a multidiscipline approach. It is not only measuring drug concentration, but also interpretation of the result. This requires knowledge of pharmacology, pharmacokinetics, genetics, pathology and Quantitative chemistry. TDM is collaborative work between physician, pharmacist, and nurse. Problems are commonly identified by physician or pharmacist. When drug monitoring request has been set, samples withdrawal is then carried out by the nurse following predetermined protocols for each drug. Sample is further processed and determined accurately using selected analytical instrument by pharmacist in laboratory.

TDM practice has been practiced for a long time globally, however, in Indonesia, the important of TDM has not been acknowledged uniformly by the physicians, even in national referral hospitals. In 2000s, one of physicians who tried to monitor patient drug level faced tiring effort and expensive cost. Nowadays, there is one private laboratory provide drug assay service, however, it seems that the service has not developed well. TDM is not a matter of drug assay, it is a series of collaborative work done by some health professionals.

\section{Drug and patient receiving TDM}

Each drug has its own pharmacokinetic profile and not all medicines require monitoring. Drugs which have narrow therapeutics window; non-liner pharmacokinetics profile, no correlation between plasma concentration and effect; high alert, high side effect/toxic and high protein binding, required to be monitored.

Drug with narrow therapeutic window, shows there is a small different between therapeutic and toxic concentration in the blood. Therefore, 
small changes in dose or patient pathophysiology, patient may not receive the effect or even lead to toxic effect. Drug with non-linier pharmacokinetics or MichaelisMenten pharmacokinetic, correlation between drug concentration and effect are difficult to predict. Similarly, drug which highly protein bind, small changes in patient status or other drug displacer, lead to toxic effect.

Therapeutic Drug Monitoring is a warrant for patients potentially altered pharmacokinetic; for examples patient with; renal, hearth or liver dysfunction, haematological disorders, diabetic and critically ill condition. TDM also required for patient receiving high alert drugs, multiple IV therapy and drugs which produce potentially interaction.

Patient who altered pharmacokinetics, dose does not correlate to effect. Critically ill patient experience unstable vital organ, therefore, TDM is required.

\section{Measuring and monitoring}

When a request of TDM has been issued. Some decision should be made for instant;

- Sampling Time

- Type of sample

- Volume of samples

- Sample container

- Sample processing

- Drug level assay

- Interpretation

Sampling time is a first step which is crucial. Sample collection cannot be withdrawn any time, it will result in wrong interpretation. Each drug has its own pharmacokinetic profile therefore, sample collection should be taken at steady state at which drug concentration in the body does not fluctuate. Steady state is commonly achieved at 4-5 times half life after starting therapy. The timing of sample withdrawal is also needed to be determined. It could be at the Tmax at when drug concentration achieved max concentration during dose interval or at when drug concentration achieves the lowest concentration. The easiest way is the last method which is called as "Trough sample collection". Sample is collected immediately before the next dose.

Type of sample used is commonly plasma, serum or whole blood. Selection to which chosen, depend on drug distribution in the blood. For example; cyclosporin distributes between erythrocytes and plasma, therefore whole blood concentration will give a reliable result. Phenytoin which highly bind to protein plasma; therefore, plasma collection will show an accurate result.

Sample volume is commonly assayed by drug concentration type, which is free drug concentration or total concentration. For highly protein bind drug, free drug concentration determination is appropriate because free drug concentration in the blood is equal to that in the receptor.

Selection of sample container or blood collecting tube is also important. There some tube contains of different anticoagulant or certain separator. For example, lithium heparin is an appropriate anticoagulant for lithium samples, while gel separator should be avoided for some drugs such as phenytoin.

Sample processes and assay are one unit. Pharmacist should select the right instrument to measure drug concentration and developing analytical method for each drug in order to produce accurate and valid result. When the result is ready to interpret, clinical pharmacist will review it and compare it to normal therapeutic range and looking at the patient condition such as laboratory result, haematology data, patient medication history, dosage regiment and the response of drug in patient. TDM result interpretation could be calculation dose adjustment, change dose interval, stopping or change other drugs and others. There has been a number of software package using Bayesian techniques, this could be useful tool in predicting of drug level.

\section{Indonesian picture}

Indonesia, is a big country. It must be many experts on this area, however, the application of TDM service has not been available yet.

The problems arise is that there is lacking of human resource who could run TDM service in hospitals. The number of pharmacists is limited in hospital. Even one of national referral hospital got a donation of TDx instrument, unfortunately, it is rarely used.

Most physician and pharmacist do not understand the principles, benefit and limitation of SDM service. Insufficient data such as 
indication of TDM, patient's history, sampling time and other information required could lead to misinterpretation.

Some health professional claims that TDM service is expensive, however the availability of this service is beneficial for the patient and the hospital. TDM service could give safer and more effective drug therapy, decrease toxicity and side effect and give better quality of life. For hospital, TDM reduces congestion, increases quality of drug therapy and service and improves the hospital performance.

\section{Reference:}

[1] PedersenL et al.2021. Evaluation of Therapeutic Drug Monitoring in the Clinical Management of patients with Rheumatic Disease: Data from a Retrospective SingleCentre Cohort Study. Biologic Targets and Therapy. V14

[2] Javadi SS etal.2018. Correlation between measured and calculated free phenytoin serum concentration in neurointensive care patients with hypoalbuminea.Clin.Pharmacol Vol.10
[3] Rianto S.2011. Therapeutic Drug Monitoring: Focus on Conditions in Indonesia. Acta Med. Indones-Indonesia J Internn Med V.43 No.3

[4] Kang JS and Lee MH. 2009. Overview of Therapeutic Drug Monitoring.KJIM.V.24

[5] Ghiculescu RA, 2008. Thepeutic drug monitoring: which drugs, why, when and how to do it,Australian Prescriber, Vol 31

[6] Annette SG. 1998. Best practice in drug monitoring. Br J Pharmacol. 46:95-99

[7] Birkett D, 1997. Pharmacokinetics made easy: Therapeutic drug monitoring, Australian Prescriber 